\title{
OSTEOPETROSIS MALIGNA INFANTIL: A PROPÓSITO DE UN CASO Y REVISIÓN DE LA LITERATURA
}

CHILD MALIGINE OSTEOPETROSIS: A PURPOSE OF A CASE AND REVIEW OF THE LITERATURE

Germán Aranzábal-Alegría1, César Espinoza-Chiong1', Dayanne Benites-Gamboa², Lino Aguirre-Retamozo³

\begin{abstract}
RESUMEN
Osteopetrosis comprende una serie de raras condiciones genéticas que produce un desequilibrio en el remodelado óseo debido a una actividad osteoclástica anormal. Reportamos una paciente mujer de 1 año 4 meses, diagnosticada de osteopetrosis maligna infantil neuropática mientras se investigaba la causa de palidez, abdomen distendido con circulación colateral y retardo en el crecimiento y de los hitos del desarrollo. Examen oftalmológico con retinopatía derecha. Radiografías del esqueleto revelaron una hiperdensidad ósea generalizada. Aspirado de medula ósea mostró hipercelularidad con la serie eritroide hiperplásica, serie blanca y megacariocítica normal. Diagnóstico fue confirmado por características clínicas y radiológicas. La paciente recibió tratamiento de soporte. Aunque el diagnostico de osteopetrosis maligna infantil es sencillo este a menudo se retrasa por la infrecuencia de la enfermedad y la falta de sospecha clínica, un diagnóstico oportuno y un tratamiento de trasplante de medula ósea son el enfoque curativo para una enfermedad de pobre pronóstico.
\end{abstract}

Palabras clave: Osteopetrosis; Infantil; Osteoclasto; Retinopatía; Hueso. (fuente: DeCS BIREME)

\begin{abstract}
Osteopetrosis comprises a series of rare genetic conditions that produce an imbalance in bone remodeling due to abnormal osteoclastic activity. We report a female patient 1 year 4 months, diagnosed with malignant osteopetrosis neuropathic child while causing paleness, distended abdomen with collateral circulation and growth retardation and developmental milestones are investigating. Examination right eye with retinopathy. Skeletal $x$-rays revealed a generalized bone hyperdensity. She bone marrow aspirate showed hypercellularity with hyperplastic erythroid, megakaryocytic normal white series. Diagnosis was confirmed by clinical and radiological features. The patient received supportive care. While the diagnosis is simple OPAR this is often delayed by the rarity of the disease and the lack of clinical suspicion, early diagnosis and treatment of bone marrow transplant is curative approach to a disease of poor prognosis.
\end{abstract}

Key words: Osteopetrosis; Infantile; Osteoclast; Retinopathy; Bone. (source: MeSH NLM)

\section{INTRODUCCIÓN}

La osteopetrosis comprende una serie de raras condiciones genéticas caracterizadas por un incremento de la densidad ósea en las radiografías', causada por una actividad osteoclástica anormal que genera un desequilibrio en la remodelación ósea ${ }^{2,3}$.
La forma autosómica dominante es usualmente asintomática frecuentemente diagnosticada en la infancia tardía o en la adultez y tiene un largo tiempo de sobrevida; sin embargo la forma autosómica recesiva, también llamada osteopetrosis maligna

\footnotetext{
${ }^{1}$ Instituto de Investigación en Ciencias Biomédicas, Universidad Ricardo Palma, Lima-Perú

${ }^{2}$ Facultad de Medicina Humana, Universidad Ricardo Palma, Lima-Perú.

${ }^{3}$ Hospital Nacional Dos de Mayo, Lima-Perú.
}

Citar como: Germán Aranzábal-Alegría, César Espinoza-Chiong, Dayanne Benites-Gamboa, Lino Aguirre-Retamozo. Osteopetrosis maligna infantil: a propósito de un caso y revisión de la literatura. Rev. Fac. Med. Hum. Octubre 2019; 19(4):126-130. DOI 10.25176/RFMH.v19i4.2345 
infantil, es diagnosticada en los primeros meses de vida al año de edad siendo esta de peor pronóstico ${ }^{4,5}$. Las manifestaciones más severas tienden a mostrarse en las formas infantiles las cuales incluyen macrocefalia con prominencias frontales, hidrocefalia, alteraciones metabólicas, anormalidades hematológicas que incluyen anemia, trombocitopenia, hematopoyesis extramedular y mayor susceptibilidad a infecciones ${ }^{6,7}$. Además, los cambios en el remodelado óseo del cráneo producen compresión nerviosa generando ceguera, sordera y parálisis facial ${ }^{8}$.

Se presenta el caso de una paciente diagnosticada de osteopetrosis maligna infantil que presenta complicaciones hematológicas y neurológicas tempranas.

\section{CASO CLÍNICO}

Paciente mujer de 1 año 4 meses de edad procedente de Oxapampa que acude a emergencia del Hospital Nacional Dos de Mayo por fiebre, congestión nasal, rinorrea, tos y baja de peso; le administraron amoxicilina más acetaminofén con la persistencia de los síntomas. Antecedente de parto eutócico de 35 semanas sin complicaciones con un peso de 2,900 gr, talla de $47 \mathrm{~cm}$ y un perímetro cefálico de $34 \mathrm{~cm}$; y al año de edad. Fue hospitalizada durante 17 días por diarrea no disentérica, desnutrición y anemia severa de presunto origen nutricional versus parasitario; además, presentó infecciones a repetición de vías respiratorias y del tracto gastrointestinal por lo que se sugirió traslado a Lima para mayores estudios.

Al examen físico presentó palidez generalizada, tejido celular subcutáneo en escasa cantidad, distiquiasis en ojo izquierdo, anisocoria, fotoreactividad conservada, hemorragia subconjuntival lateral en ambos ojos, fontanela anterior de $4 \mathrm{~cm}$, congestión nasal derecha, soplo sistólico multifocal II/ $\mathrm{V}$, roncantes difusos bilaterales, abdomen distendido con circulación colateral y hepatomegalia $(7 \mathrm{~cm}$ del reborde subcostal de consistencia normal). Se observó retardo severo del crecimiento y de los hitos del desarrollo (no control cervico-torácico, lenguaje con monosílabos). Además de presentar hipoacusia bilateral.

Los exámenes auxiliares mostraron lo siguiente: $\mathrm{Hb}$ 4,5 gr/dl, leucocitos 22, 287 con predominio de segmentados $(11,145 / \mathrm{mm} 3)$, conteo de plaquetas $20,000 / \mathrm{mm} 3$, linfocitos atípicos $20 \%$, blastos $10 \%$.
PCR 17,30 mg\%, VSG 50 ml/h, Examen bioquímico TTP 47,6 segundos, LDH 2053 UI/l, AFP 9,79 ng/m, Calcio Sérico 8,7 mg/dl, TSH 1,32 $\mu \mathrm{g}$, T4 0,7 $\mu \mathrm{g}$, PTH 32,12 $\mathrm{pg} / \mathrm{ml}$, resto de exámenes dentro de los parámetros normales. Examen de orina con hematíes 12-15 / campos. Electroforesis de hemoglobina normal. Test de Coombs directo, serología para TORCH, ELISA VIH y Hepatitis fue negativa.

La radiografía de tórax, base cráneo y miembros inferiores mostraron una hiperdensidad ósea generalizada (Figura 1, 2, 3 y 4). Sin embargo, la densitometría ósea de columna lumbar se encontró en rangos normales; la ultrasonografía abdominal detectó hepatomegalia marcada y la cardiaca no demostró alteración estructural alguna. La tomografía espiral multicorte abdominopélvica demostró hepatomegalia con probable esteatosis, no adenomegalias y constipación. A la oftalmoscopía se evidenció neuropatía óptica y retinopatía del ojo derecho. Aspirado medular mostró hipercelularidad, relación $\mathrm{M} / \mathrm{E}: 1 / 1$, serie eritroide hiperplasia severa con predominio ortocromático, serie mieloide normal con detención en su maduración con blastos de 4,5\%, serie megacariocítica normal, serie linfoide $8 \%$ y células plasmáticas $0 \%$. Estudio citogenético normal. No se realizó potenciales evocados auditivos debido a equipo mal calibrado al momento.

Dentro de los diagnósticos diferenciales planteados fueron los síndromes mielodisplásicos, enfermedad de Paget en su forma ósea, hipoparatiroidismo, pseudohipoparatiroidismo e infecciones congénitas. El diagnóstico definitivo fue una osteopetrosis maligna infantil en la variante neuropática por las correlaciones de las características radiográficas y clínicas; sin embargo, no se pudo realizar un análisis genético dados los escasos recursos de familiares. La paciente recibió tratamiento de soporte y sintomático incluyendo terapia antibiótica para neumonía intrahospitalaria por Staphylococcus epidermidis, eritropoyetina, transfusiones sanguíneas y suplementación de vitamina $D$ y calcio. La paciente fue dada de alta a los 3 meses de hospitalización con referencia al Instituto Nacional del Niño para un trasplante de médula ósea, pero a los ocho días tuvo un reingreso por síndrome de obstrucción bronquial con resolución favorable, no se realizó seguimiento al caso debido al traslado a otro establecimiento de salud. 


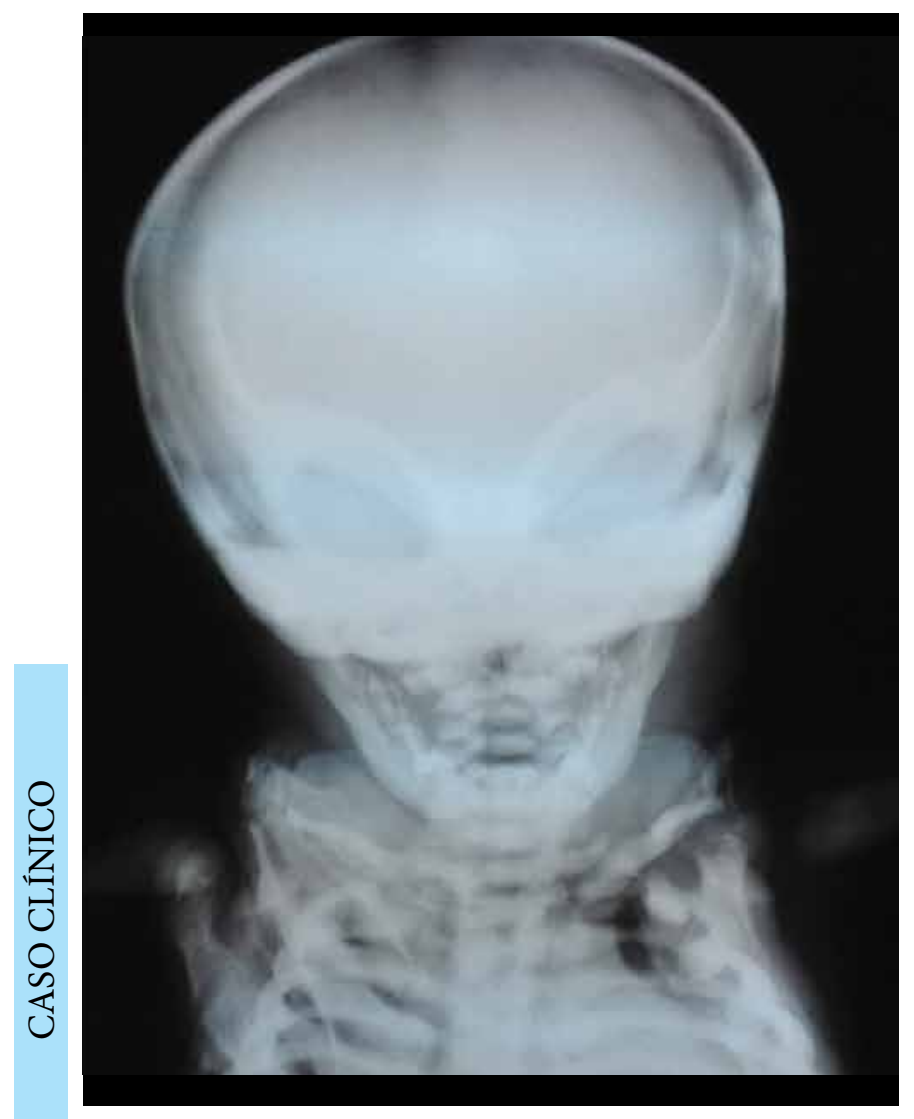

Figura 1. Radiografía esqueleto facial. Note las esclerosis de las orbitas y hueso esfenoides como "apariencia de máscara de Arlequín".

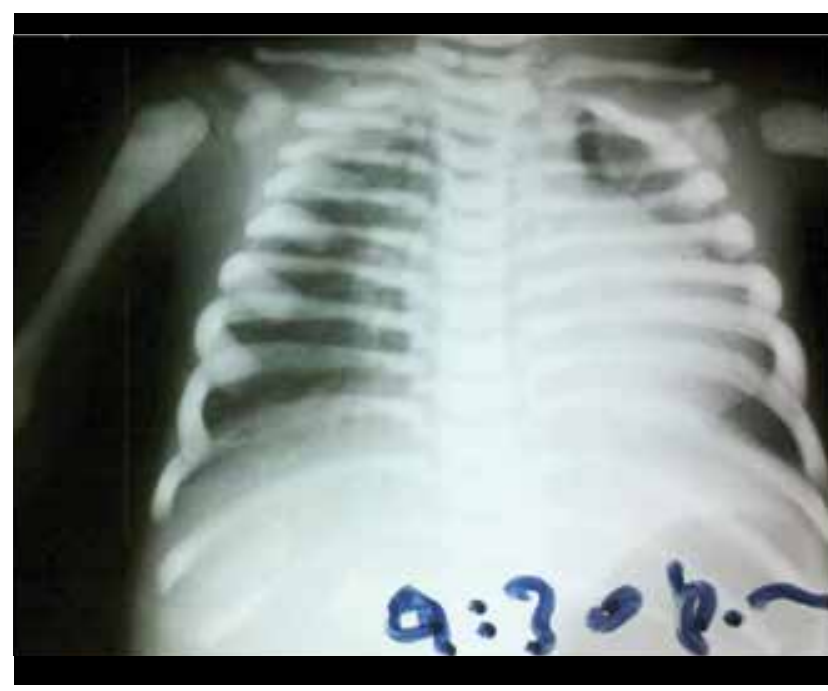

Figura 2. Radiografía de Tórax. Note el rosario raquítico.

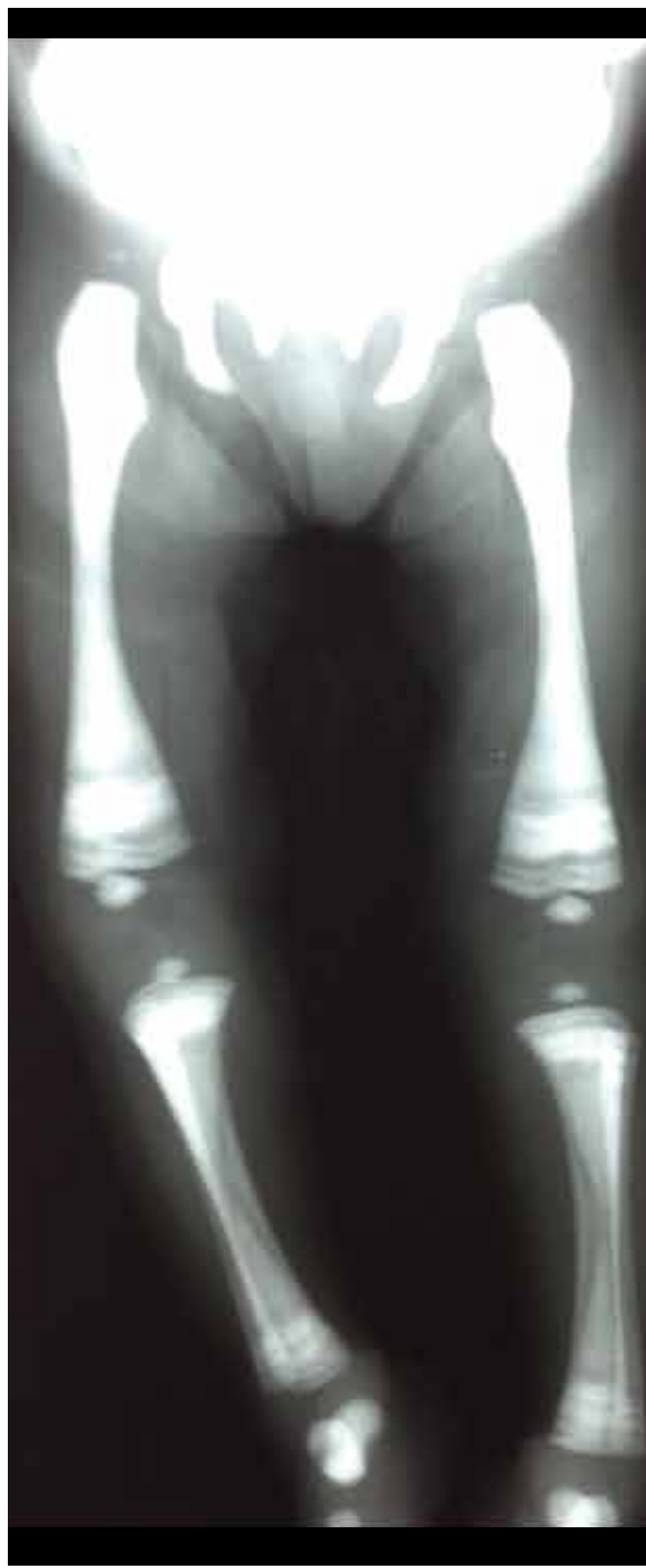

Figura 3. Radiografía de pelvis y miembros inferiores. Note la densidad ósea generalizada con apariencia "hueso dentro del hueso" o la deformidad ósea en matraz de Erlenmeyer, reconocible con facilidad en el fémur y piernas en varo. 


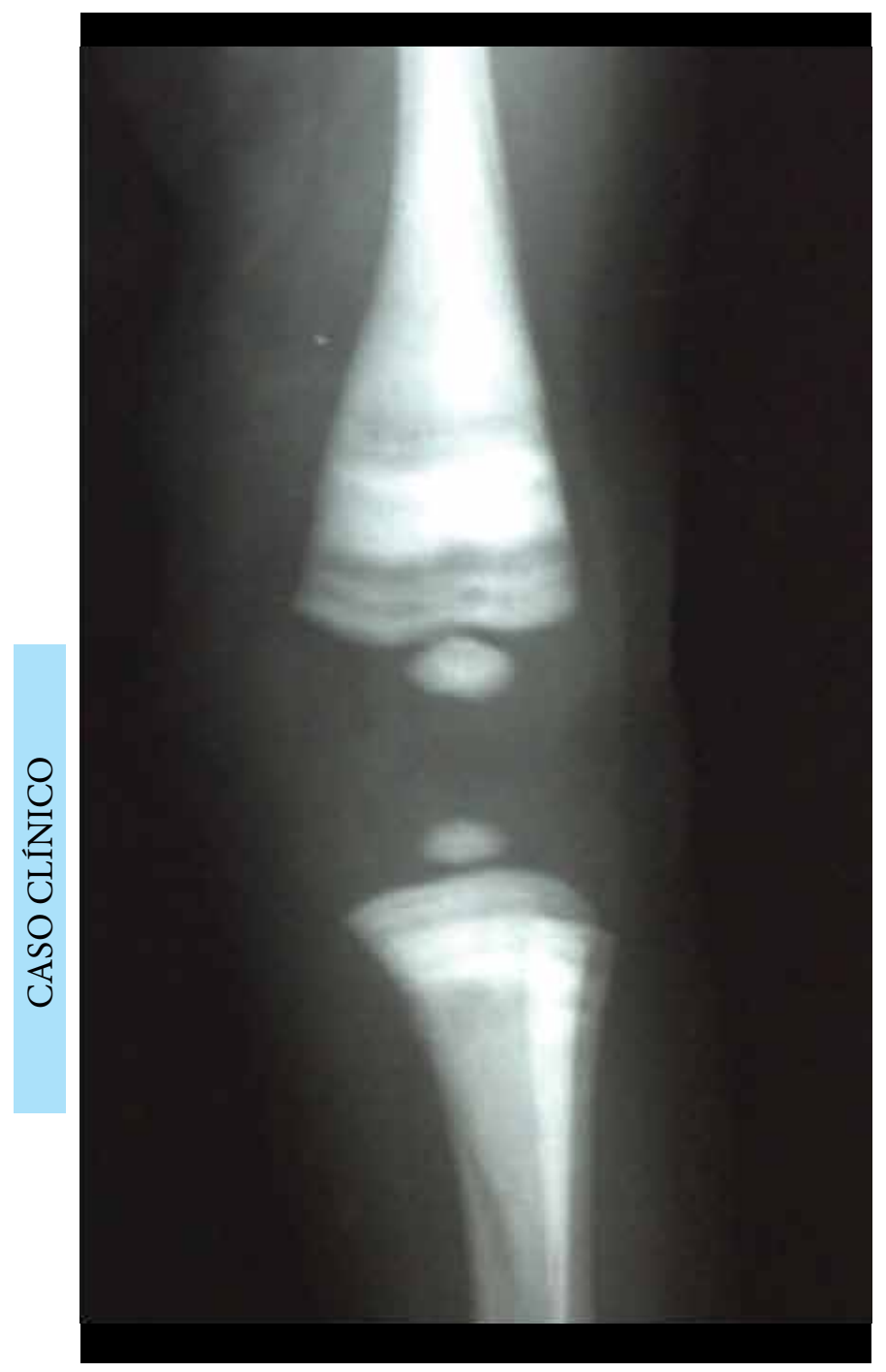

Figura 4. Radiografía distal fémur y tibial proximal izquierdo. Note los defectos del modelado metafisiario y las características bandas luminosas.

\section{DISCUSIÓN}

La osteopetrosis (OP) , también llamada enfermedad de Albers-Schönberg o enfermedad de los huesos marmóleos ${ }^{8}$, comprende a un conjunto de enfermedades clínicamente diferentes pero que comparten la misma característica radiológica de un aumento de la densidad ósea-11.

Esta rara patología carece de datos epidemiológicos precisos, pero se estima que la incidencia es menos de 1-3,4:200,000 nacimientos según Del Fatorre et $\mathrm{al}^{12}$. Su etiología es desconocida aunque en modelos animales fue asociado a una alteración en la formación y maduración de los osteoclastos que se vuelven incapaces de realizar la reabsorción ósea y cartilaginosa generando un desequilibrio en el remodelado óseo ${ }^{13}$. Los tipos de OP varían de acuerdo a su severidad, se dividen en dos grandes grupos: 1.OP Autosómico recesivos (OPAR), 2.OP Autosómico dominantes (OPAD) y 3. Ligado al cromosoma $X^{3}$.
El diagnóstico de la OP se realiza principalmente por los hallazgos clínico-laboratoriales siendo los hallazgos radiológicos los que nos dan el diagnóstico 8 , pues no existe una prueba específica para el mismo $^{12}$. La variedad OPAR se presenta clásicamente durante los primeros meses de vida ${ }^{14}$, como es el caso de nuestro paciente. Además existe una predisposición a padecer complicaciones como tupidez nasal relacionada a malformación de los senos paranasales, neuropatías por compresión nerviosa y pancitopenia ${ }^{8}$, evidenciadas en la historia clínica de la paciente. Las características clínicas de la OPAR son las siguientes: alteraciones en el crecimiento, macrocefalia asociada a hidrocefalia y prominencia frontal, estenosis de las coanas, alteraciones de los senos paranasales, alteraciones hematológicas compatibles con aplasia medular, alteraciones neurológicas como ceguera, sordera y parálisis facial asociada a una estenosis del foramen por donde se conducen estos nervios y defectos odontológicos caracterizados por la presencia de caries severa ${ }^{14}$ De las características clínicas mencionadas, nuestro paciente presentó una alteración en el crecimiento y de los hitos desarrollo, además de alteración neurológica como hipoacusia bilateral, neuropatía óptica y retinopatía derecha. Los exámenes auxiliares mostraron anemia, trombocitopenia, leucocitosis con desviación izquierda, blastos $10 \%$ y hepatomegalia descartándose un origen mielodisplásico. Estudios reportan el enmascaramiento y la asociación de la osteopetrosis maligna infantil y las leucemias; sin embargo, en el aspirado de medula ósea de nuestra paciente no se encontraron hallazgos compatibles con esta ${ }^{15}$. Las características radiográficas típicas de la osteopetrosis incluyen un marcado incremento de la densidad ósea, que puede ser observado en la figura 1,2,3 y 4 pero en los informes iniciales de radiología de nuestro paciente fueron catalogadas como normales, provocando un retraso en el diagnóstico. En la OPAR existen subtipos como son: 1. OPAR neuropática, 2.OPAR más acidosis tubular renal ${ }^{15}$, por la clínica que presento nuestro paciente se encuentra en la variedad neuropática caracterizada por neuropatía e hipoacusia a pesar de no realizarse una evaluación neurofisiológica de la audición. Es importante hacer esta diferencia pues cada tipo tiene una respuesta distinta al tratamiento y grado de riego de recaídas ${ }^{8}$.

Actualmente no se cuenta con un tratamiento eficaz en contra de esta patología ${ }^{8}$, el manejo ofrecido al paciente es de brindarle soporte de acuerdo a la sintomatología que va presentando ${ }^{11}$ durante el curso natural de la enfermedad, a nuestro paciente se le 
ofreció un tratamiento para la infección por neumonía intrahospitalaria que fue el motivo de hospitalización prolongada y tratado con antibióticos durante 14 días mostrando mejoría, además al existir una disminución de la serie roja y megacariocítica, se le comenzó a administrar hematinicos con los cuales fue dado de alta.

A pesar de tener un conocimiento de la fisiopatología aún no se puede precisar la etiología de esta enfermedad además de un tratamiento para evitar la severidad de los síntomas como neuropatía por compresión del nervio óptico y vestíbulo-coclear9. La OP tiene un pronóstico asociado a la severidad del cuadro, en las formas infantiles los niños fallecen como resultado de la supresión de la medula ósea ${ }^{6}$ e infecciones recurrentes. Actualmente el trasplante de progenitores hematopoyéticos representa una alternativa terapéutica cuya respuesta dependerá del tipo de mutación genética subyacente ${ }^{5}$. A pesar que el diagnóstico de la osteopetrosis maligna infantil es sencillo y depende de un examen radiológico y clínicolaboratorial, este a menudo se retrasa debido a la rareza de la enfermedad y a la falta sospecha clínica, según Essabar et $\mathrm{al}^{4}$, los estudios genéticos tienen relevancia para dar consejería genética a los progenitores de los pacientes aquejados por osteopetrosis, pero no fueron llevados a cabo para nuestra paciente, dado su elevado costo. El diagnóstico temprano y tratamiento de soporte oportuno prolonga la vida de estos pacientes que de otra forma resultaría fatal. Se sugiere realizar mayor cantidad de estudios en animales para poder descubrir nuevos blancos terapéuticos que mejoren la calidad de vida de los pacientes.

\section{Agradecimientos}

Francisca Valero-Villaízan, por su apoyo en la búsqueda bibliográfica.

Contribuciones de autoría: Espinoza-Chiong C, Aranzábal-Alegría Gy Benites-Gamboa D; contribuyeron en redacción y revisión final del caso clínico, AguirreRetamozo L contribuyó en la revisión final del caso clínico.

\section{Financiamiento: Autofinanciado.}

Conflicto de interés: Los autores declaran no tener conflictos de interés respecto al contenido de la publicación de este caso clínico.

Recibido: 27 de junio del 2019

Aprobado: 16 de setiembre del 2019

Correspondencia: German Aranzábal-Alegría

Dirección: Avenida Colombia 247, Lima 21, Perú.

Teléfono: 984767905

Correo:garanzabal456@gmail.com

\section{REFERENCIAS BIBLIOGRÁFICAS}

1. Mejía Osuna P, Santos-Guzmán J, Villela L, Cedillo-Alemán EJ, García A. Osteopetrosis, calcificación más allá del sistema óseo. Reporte de un caso. Bol Méd Hosp Infant México. abril de 2012;69(2):116-20. Disponible en: http://www.scielo.org.mx/scielo.php?script=sci arttext\&pid=S1665-11462012000200007

2. Janer Subías E, de Arriba Muñoz A, García Iñiguez JP, Ferrer Lozano M, Sanchez del Pozo J, Labarta Aizpun Jl. Osteopetrosis autosómica dominante: a propósito de 3 casos y una mutación. An Pediatría. 1 de enero de 2015;82(1): e35-8. Disponible en: https://www.sciencedirect com/science/article/abs/pii/S1695403314001623

3. Essabar L, Meskini T, Ettair S, Erreimi N, Mouane N. Malignant infantile osteopetrosis: case report with review of literature. Pan Afr Med [Internet]. 27 de 2014 [citado 2 de septiembre de 2019];17. Disponible en: http://www.panafrican-med-journal.com/content/article/17/63/ full/

4. Kant P, Sharda N, Bhowate RR. Clinical and Radiological Findings of Autosomal Dominant Osteopetrosis Type II: A Case Report [Internet] Case Reports in Dentistry. 2013 [citado 2 de septiembre de 2019]. Disponible en: https://www.hindawi.com/journals/crid/2013/707343/

5. Lam DK, Sandor GKB, Holmes HI, Carmichael RP, Clokie CML. Marble Bone Disease: A Review of Osteopetrosis and Its Oral Health Implications for Dentists. J Can Dent Assoc. 2007;73(9):5. Disponible en: http://www. cda-adc.ca/jcda/vol-73/issue-9/839.html

6. Balemans W, Van Wesenbeeck L, Van Hul W. A clinical and molecular overview of the human osteopetroses. Calcif Tissue Int. 2005;77(5):263-74. Disponible en: https://link.springer.com/ article/10.1007\%2Fs00223-005-0027-6

7. Chen C-J, Lee M-Y, Hsu M-L, Lien S-H, Cheng S-N. Malignant infantile osteopetrosis initially presenting with neonatal hypocalcemia: case report. Ann Hematol. 2003;82(1):64-7. Disponible en: https://www. ncbi.nlm.nih.gov/pubmed/12574970
8. Stark Z, Savarirayan R. Osteopetrosis. Orphanet J Rare Dis. 2009;4:5. Disponible en: https://www.ncbi.nlm.nih.gov/pmc/articles/ PMC2654865/

9. Engiz O, Kara S, Bagrul D, Lahr G, Alioglu B, Arikan I, et al. Infantile malignant osteopetrosis: a rare cause of neonatal hypocalcemia. J Pediatr Endocrinol Metab JPEM. 2012;25(11-12):1205-7. Disponible en: https://www.researchgate.net/publication/235700509 Infantile malignant_osteopetrosis_A_rare_cause_of_neonatal_hypocalcemia

10. Rao P. T, V. S, T.P. G, Harsha S. Infantile or Malignant Osteopetrosis Case Report of Two Siblings. J Clin Diagn Res JCDR. 2013;7(8):175960. Disponible en: https://www.ncbi.nlm.nih.gov/pmc/articles/ PMC3782963/

11. Kurt A, Sen Y, Elkiran O, Akarsu S, Kurt ANC, Aygun AD. Malignant infantile osteopetrosis: a rare cause of neonatal hypocalcemia. J Pediatr Endocrinol Metab JPEM. 2006;19(12):1459-62. Disponible en: https:// www.ncbi.nlm.nih.gov/pubmed/17252700

12. Del Fattore A, Cappariello A, Teti A. Genetics, pathogenesis and complications of osteopetrosis. Bone. enero de 2008;42(1):19-29. Disponible en: https://www.sciencedirect.com/science/article/abs/pii/ S8756328207006278?via\%3Dihub

13. Blin-Wakkach C, Wakkach A, Sexton PM, Rochet N, Carle GF Hematological defects in the oc/oc mouse, a model of infantile malignant osteopetrosis. Leukemia. septiembre de 2004;18(9):1505-11. Disponible en: https://www.nature.com/articles/2403449

14. Kumar KJ, Bandaru K, Prashanth SN, Mamatha S. Malignant infantile osteopetrosis. Indian J Hum Genet. enero de 2013;19(1):90-2. Disponible en: https://www.ncbi.nlm.nih.gov/pmc/articles/PMC3722638/

15. Kocher MS, Kasser JR. Osteopetrosis. Am J Orthop Belle Mead NJ. mayo de 2003;32(5):222-8. Disponible en: https://www.ncbi.nlm.nih. gov/pubmed/12772872 